\title{
Article \\ ESBL Activity, MDR, and Carbapenem Resistance among Predominant Enterobacterales Isolated in 2019
}

\author{
Altaf Bandy ${ }^{1, *(D)}$ and Bilal Tantry ${ }^{2}$ (D) \\ 1 Family \& Community Medicine, College of Medicine, Jouf University, 74311 Sakaka, Aljouf, Saudi Arabia \\ 2 Ex-Faculty, Department of Microbiology, College of Medicine, Jouf University, \\ 74311 Sakaka, Aljouf, Saudi Arabia; bilaltantry@gmail.com \\ * Correspondence: drbanday@gmail.com or ahbandy@ju.edu.sa
}

check for

updates

Citation: Bandy, A.; Tantry, B. ESBL Activity, MDR, and Carbapenem Resistance among Predominant Enterobacterales Isolated in 2019. Antibiotics 2021, 10, 744. https:// doi.org/10.3390/antibiotics10060744

Academic Editor: Cécile Muller

Received: 14 May 2021

Accepted: 16 June 2021

Published: 19 June 2021

Publisher's Note: MDPI stays neutral with regard to jurisdictional claims in published maps and institutional affiliations.

Copyright: (c) 2021 by the authors. Licensee MDPI, Basel, Switzerland. This article is an open access article distributed under the terms and conditions of the Creative Commons Attribution (CC BY) license (https:/ / creativecommons.org/licenses/by/ $4.0 /)$.

\begin{abstract}
Antimicrobial-resistance in Enterobacterales is a serious concern in Saudi Arabia. The present study retrospectively analyzed the antibiograms of Enterobacterales identified from 1 January 2019 to 31 December 2019 from a referral hospital in the Aljouf region of Saudi Arabia. The revised document of the Centers for Disease Control (CDC) CR-2015 and Magiorakos et al.'s document were used to define carbapenem resistance and classify resistant bacteria, respectively. The association of carbapenem resistance, MDR, and ESBL with various sociodemographic characteristics was assessed by the chi-square test and odds ratios. In total, 617 Enterobacterales were identified. The predominant $(n=533(86.4 \%))$ isolates consisted of $232(37.6 \%), 200(32.4 \%)$, and 101 (16.4\%) Escherichia coli, Klebsiella pneumoniae, and Proteus mirabilis, respectively. In general, 432 (81.0\%) and $128(24.0 \%)$ isolates were of MDR and ESBL, respectively. The MDR strains were recovered in higher frequency from intensive care units $(\mathrm{OR}=3.24(1.78-5.91) ; p<0.01)$. E. coli and K. pneumoniae resistance rates to imipenem (2.55 (1.21-5.37); $p<0.01)$ and meropenem (2.18 (1.01-4.67); $p<0.04)$, respectively, were significantly higher in winter. The data emphasize that MDR isolates among Enterobacterales are highly prevalent. The studied Enterobacterales exhibited seasonal variation in antimicrobial resistance rates towards carbapenems and ESBL activity.
\end{abstract}

Keywords: Enterobacterales; E. coli; K. pneumoniae; P. mirabilis; antimicrobials; multidrug resistance; carbapenem; ESBL

\section{Introduction}

Globally, infections with resistant Gram-negative bacteria are recognized as a severe threat to patients' health. Enterobacteriaceae resistant to third-generation cephalosporins and carbapenems are critical microorganisms that require urgent attention [1]. A greater number of patients are at risk of dying from infections caused by carbapenem-resistant, extended-spectrum beta-lactamase (ESBL)-producing, and multidrug-resistant (MDR) Enterobacteriacea [2]. Carbapenemase-producing Enterobacteriaceae infections are associated with a very high case fatality rate with currently available antibiotics [3,4]. Intrinsic and acquired bacterial resistance, international travel, inappropriate antibiotic use, and poor infection control activities are predisposing factors for developing resistance $[5,6]$.

Global dissemination of resistant Enterobacteriaceae is increasing [7,8]. The Kingdom of Saudi Arabia receives numerous pilgrims throughout the year, particularly in the holy month of Hajj, raising the possibility of resistant Enterobacteriaceae transmission. International travel is a serious concern for the transmission of resistant Enterobacteriaceae in the country [9]. Various studies and reports emerging from different regions of the kingdom have highlighted the spread of such resistant strains [10-14]. Furthermore, the expatriate population in the kingdom is a matter of concern considering the transmission of Enterobacteriaceae with diverse resistance mechanisms [15].

The surveillance of resistant strains is a prerequisite for control measures [16]. Insights into antibiotic resistance provide valuable information about significant pathogens 
and guide actions of antimicrobial stewardship programs, infection control committees, and public health agencies [17]. No exclusive study has reported antimicrobial resistance in Enterobacterales from the Aljouf region. The present study was conducted to determine carbapenem resistance, ESBL activity, and multidrug resistance among predominant Enterobacterales from a referral hospital in the Aljouf region of Saudi Arabia.

\section{Results}

\subsection{Enterobacterales and Associated Sociodemographic Characteristics}

Of the 617 Enterobacterale isolates, 232 (37.6\%), 200 (32.4\%), and $101(16.4 \%)$ isolates were of E. coli, K. pneumoniae, and P. mirabilis, respectively. The other less frequent Enterobacterales included 27 (4.4\%) Enterobacter aerogenes (Klebsiella aerogenes), 22 (3.6\%) Enterobacter cloacae (Klebsiella cloacae), 15 (2.4\%) Providencia stuartii, 13 (2.1\%) Serratia marcescens, and $7(1.1 \%)$ Morganella morganii. Further analysis of the 533 predominant Enterobacterales revealed that various sociodemographic characteristics such as sex, specimen type, referring unit, and resistance type were significantly $(p<0.05)$ associated with the distribution of E. coli, K. pneumoniae, and P. mirabilis. In general, $432(81.0 \%), 97(18.2 \%)$, and $15(2.8 \%)$ isolates were $\mathrm{MDR}, \mathrm{XDR}$, and $\mathrm{PDR}$, respectively. Of the 432 multidrug-resistant isolates, $167(38.6 \%), 170(39.3 \%)$, and $95(21.9 \%)$ isolates were E. coli, K. pneumoniae, and P. mirabilis, respectively. Furthermore, $128(24.0 \%)$ of these Enterobacterales were extended-spectrum beta-lactamase (ESBL) producers (Table 1).

Table 1. Distribution characteristics and resistance profile in E. coli, K. pneumoniae, and P. mirabilis $(n=533)$.

\begin{tabular}{|c|c|c|c|c|c|}
\hline \multicolumn{2}{|c|}{ Characteristic } & \multirow{2}{*}{$\frac{\text { E. coli (232) }}{89}$} & \multirow{2}{*}{$\frac{\text { K. pneumoniae (200) }}{118}$} & \multirow{2}{*}{$\frac{\text { P. mirabilis (101) }}{59}$} & \multirow{3}{*}{$\begin{array}{c}\boldsymbol{p} \text { Value } \\
0.01\end{array}$} \\
\hline & Male & & & & \\
\hline Sex & Female & 143 & 82 & 42 & \\
\hline \multirow{4}{*}{ Age group (years) } & $\geq 60$ & 100 & 97 & 51 & \multirow{4}{*}{0.05} \\
\hline & $40-59$ & 39 & 51 & 21 & \\
\hline & $20-39$ & 68 & 37 & 20 & \\
\hline & $\leq 19$ & 25 & 15 & 9 & \\
\hline \multirow{4}{*}{ Seasonality } & Quarter 1 & 75 & 85 & 37 & \multirow{4}{*}{0.37} \\
\hline & Quarter 2 & 55 & 47 & 21 & \\
\hline & Quarter 3 & 44 & 28 & 20 & \\
\hline & Quarter 4 & 58 & 40 & 23 & \\
\hline \multirow{3}{*}{ * Specimen type } & Urine & 161 & 97 & 38 & \multirow{3}{*}{0.01} \\
\hline & Blood & 23 & 63 & 24 & \\
\hline & Wound swab & 48 & 40 & 8 & \\
\hline \multirow{4}{*}{ ** Referring unit } & Intensive care units & 63 & 120 & 61 & \multirow{4}{*}{0.01} \\
\hline & Outpatient departments & 64 & 29 & 21 & \\
\hline & Surgical wards & 45 & 27 & 9 & \\
\hline & Medical wards & 24 & 10 & 10 & \\
\hline \multirow{4}{*}{ *** Resistance Type } & MDR & 167 & 170 & 95 & \multirow{4}{*}{0.01} \\
\hline & XDR & 8 & 65 & 24 & \\
\hline & PDR & 2 & 12 & 1 & \\
\hline & ESBL & 85 & 26 & 17 & \\
\hline
\end{tabular}

* From tracheal wash (8) and sputum (23) samples P. mirabilis was the only isolate, not included in analysis. ** P. mirabilis was not isolated from specimens referred from other hospitals, not included in analysis. ${ }^{* * *}$ Yates correction.

\subsection{MDR, ESBL, and Associated Sociodemographic Characteristics}

The multidrug-resistant organisms were isolated with significantly higher frequency from clinical samples of intensive care units $(\mathrm{OR}=3.24(1.78-5.91) ; p<0.01)$ compared to the samples received from surgical wards and other hospitals $(\mathrm{OR}=2.67(1.29-5.50)$; $p<0.01)$. Other sociodemographic characteristics did not reveal any significant association with multidrug strains (Table 2). 
Table 2. Sociodemographic distribution characteristics of multidrug-resistant isolates $(n=432)$.

\begin{tabular}{|c|c|c|c|c|c|}
\hline \multicolumn{2}{|c|}{ Characteristic } & \multirow{2}{*}{$\frac{\text { MDR }}{213}$} & \multirow{2}{*}{$\begin{array}{c}\text { Non-MDR } \\
53\end{array}$} & \multirow{3}{*}{$\begin{array}{c}\text { OR }(\mathbf{9 5} \% \mathbf{C I}) \\
0.88 \\
(0.57-1.35)\end{array}$} & \multirow{3}{*}{$\begin{array}{c}\boldsymbol{p} \text { Value } \\
0.64\end{array}$} \\
\hline & Male & & & & \\
\hline Sex & Female & 219 & 48 & & \\
\hline & $\geq 60$ & 204 & 44 & 1 & \\
\hline \multirow[t]{4}{*}{ Age group (years) } & $40-59$ & 89 & 22 & $\begin{array}{c}1.14 \\
(0.65-2.02)\end{array}$ & 0.64 \\
\hline & 20-39 & 96 & 29 & $\begin{array}{c}1.40 \\
(0.82-2.37)\end{array}$ & 0.21 \\
\hline & $\leq 19$ & 43 & 6 & $\begin{array}{c}0.64 \\
(0.25-1.61)\end{array}$ & 0.46 \\
\hline & Quarter 1 & 155 & 42 & 1 & \\
\hline \multirow[t]{4}{*}{ Seasonality } & Quarter 2 & 101 & 22 & $\begin{array}{c}0.80 \\
(0.45-1.42)\end{array}$ & 0.54 \\
\hline & Quarter 3 & 72 & 20 & $\begin{array}{c}1.02 \\
(0.56-1.87)\end{array}$ & 0.92 \\
\hline & Quarter 4 & 104 & 17 & $\begin{array}{c}0.60 \\
(0.32-1.11)\end{array}$ & 0.14 \\
\hline & Urine & 238 & 58 & 1 & \\
\hline \multirow[t]{3}{*}{ * Specimen type } & Blood & 82 & 12 & $\begin{array}{c}0.60 \\
(0.30-1.17)\end{array}$ & 0.17 \\
\hline & Wound swab & 81 & 31 & $\begin{array}{c}1.57 \\
(0.94-2.59)\end{array}$ & 0.10 \\
\hline & Intensive care units & 213 & 31 & 1 & \\
\hline \multirow[t]{4}{*}{ Referring unit } & Outpatient departments & 92 & 22 & $\begin{array}{c}1.64 \\
(0.90-2.98)\end{array}$ & 0.10 \\
\hline & Surgical wards & 55 & 26 & $\begin{array}{c}3.24 \\
(1.78-5.91)\end{array}$ & 0.01 \\
\hline & Medical wards & 36 & 8 & $\begin{array}{c}1.52 \\
(0.65-3.58)\end{array}$ & 0.46 \\
\hline & Referred from other hospitals & 36 & 14 & $\begin{array}{c}2.67 \\
(1.29-5.50)\end{array}$ & 0.01 \\
\hline
\end{tabular}

*P. mirabilis was the only Enterobacterale isolated from tracheal wash (8) and sputum (23) samples, not included in analysis.

The ESBL isolates were observed with significantly $(p<0.05)$ lesser frequency in the samples received in the second, third, and fourth quarters of the year compared to the first quarter (January-March) (Table 3).

\subsection{Carbapenem Resistance and Associated Sociodemographic Characteristics}

K. pneumoniae exhibited $62.5 \%, 62 \%$, and $58.3 \%$ resistance towards ertapenem, imipenem, and meropenem, respectively. A resistance rate of $26.5 \%$ towards meropenem was observed in P. mirabilis.

Meropenem resistance in $P$. mirabilis was significantly higher among the male patients (5.5 (1.72-17.56); $p<0.01)$ and patients aged $\geq 50$ years $(4.15(1.29-13.30) ; p<0.02)$. In E. coli and $K$. pneumoniae, seasonal variation in the antimicrobial resistance rate was observed for imipenem (2.55 (1.21-5.37); $p<0.01)$ and meropenem (2.18 (1.01-4.67); $p<0.04)$, respectively. Furthermore, the K. pneumoniae meropenem resistance rate was significantly higher in samples received from intensive care units than from other units $(1.92(1.08-3.43) ; p<0.02)$ (Table 4). 
Table 3. Sociodemographic distribution characteristics of ESBL isolates $(n=128)$.

\begin{tabular}{|c|c|c|c|c|c|}
\hline \multicolumn{2}{|c|}{ Characteristic } & \multirow{2}{*}{$\begin{array}{c}\text { ESBL } \\
61\end{array}$} & \multirow{2}{*}{$\begin{array}{c}\text { Non-ESBL } \\
205\end{array}$} & \multirow{3}{*}{$\begin{array}{c}\text { OR }(\mathbf{9 5} \% \mathbf{C I}) \\
0.88 \\
(0.59-1.32)\end{array}$} & \multirow{3}{*}{$\begin{array}{c}p \text { Value } \\
0.55\end{array}$} \\
\hline & Male & & & & \\
\hline Sex & Female & 67 & 200 & & \\
\hline & $\geq 60$ & 67 & 181 & 1 & \\
\hline \multirow[t]{4}{*}{ Age group (years) } & $40-59$ & 21 & 90 & $\begin{array}{c}1.58 \\
(0.91-2.75)\end{array}$ & 0.09 \\
\hline & 20-39 & 28 & 97 & $\begin{array}{c}1.28 \\
(0.77-2.15)\end{array}$ & 0.19 \\
\hline & $\leq 19$ & 12 & 37 & $\begin{array}{c}1.14 \\
(0.56-2.31)\end{array}$ & 0.71 \\
\hline & Quarter 1 & 32 & 165 & 1 & \\
\hline \multirow[t]{4}{*}{ Seasonality } & Quarter 2 & 35 & 88 & $\begin{array}{c}0.48 \\
(0.28-0.84)\end{array}$ & 0.01 \\
\hline & Quarter 3 & 25 & 67 & $\begin{array}{c}0.51 \\
(0.28-0.94)\end{array}$ & 0.02 \\
\hline & Quarter 4 & 36 & 85 & $\begin{array}{c}0.45 \\
(0.26-0.78)\end{array}$ & 0.01 \\
\hline & Urine & 70 & 226 & 1 & \\
\hline \multirow[t]{3}{*}{ * Specimen type } & Blood & 26 & 68 & $\begin{array}{c}0.81 \\
(0.47-1.37)\end{array}$ & 0.43 \\
\hline & Wound swab & 23 & 89 & $\begin{array}{c}1.19 \\
(0.70-2.03)\end{array}$ & 0.50 \\
\hline & Intensive care units & 49 & 195 & 1 & \\
\hline \multirow[t]{4}{*}{ Referring unit } & Outpatient departments & 24 & 90 & $\begin{array}{c}0.94 \\
(0.54-1.63)\end{array}$ & 0.82 \\
\hline & Surgical wards & 21 & 60 & $\begin{array}{c}0.71 \\
(0.39-1.29)\end{array}$ & 0.26 \\
\hline & Medical wards & 18 & 26 & $\begin{array}{c}0.36 \\
(0.18-0.71)\end{array}$ & 0.01 \\
\hline & Referred from other hospitals & 16 & 34 & $\begin{array}{c}0.53 \\
(0.27-1.04)\end{array}$ & 0.06 \\
\hline
\end{tabular}

*P. mirabilis was the only Enterobacterale isolated from tracheal wash (5) and sputum (4) samples, not included in analysis.

\subsection{Antimicrobial Resistance among Predominant Enterobacterales}

Aminoglycosides (amikacin and gentamycin) are effective antibiotics against $E$. coli and K. pneumoniae. E. coli and K. pneumoniae exhibited sensitivities of $96.5 \%$ and $74.9 \%$ towards amikacin, respectively. First-generation cephalosporin (cephalothin) was ineffective, and a resistance rate of $>80 \%$ was observed. All the studied bacteria presented $>50 \%$ resistance to second- through fourth-generation cephalosporins, except for cefoxitin. Third-generation cephalosporins were less effective against K. pneumoniae ( $27.4 \%$ highest for ceftriaxone) and P. mirabilis (25.0\% highest for ceftazidime). P. mirabilis exhibited highest resistance $(76.8 \%)$ towards cefepime (fourth-generation cephalosporin) (Table 5).

The meropenem multidrug-resistant (carbapenem-resistant Enterobacteriaceae) strains of P. mirabilis and E. coli exhibited the highest $(100.0 \%)$ and lowest $(47.0 \%)$ resistance rates to tigecycline, respectively. Almost all $(96.0 \%)$ of the tested meropenem multidrug-resistant strains of P. mirabilis were resistant to colistin (Figure 1). 
Table 4. Carbapenem resistance distribution characteristics of predominant Enterobacterales.

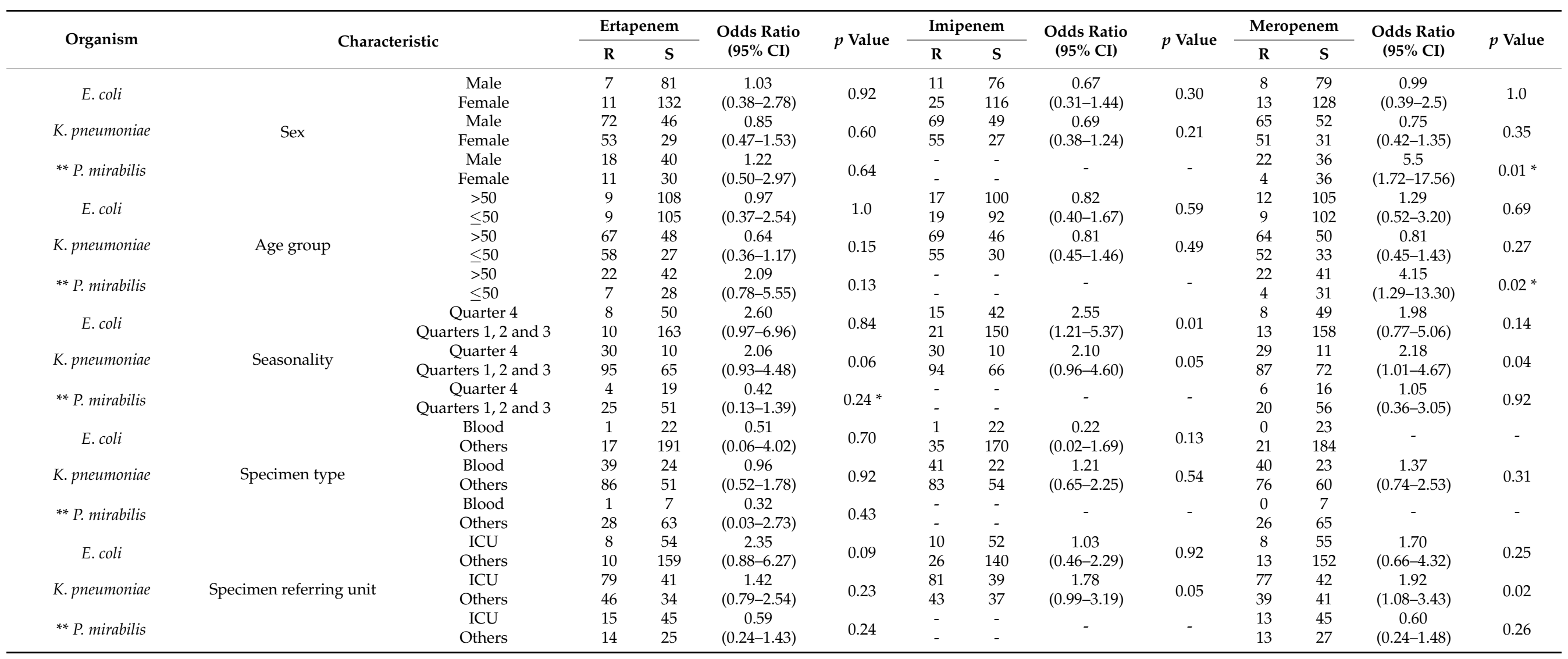

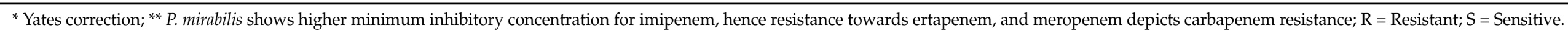


Table 5. Antimicrobial sensitivity of the studied organisms.

\begin{tabular}{cccc}
\hline Antibiotic & E. coli $\mathbf{( \% )}$ & K. pneumoniae (\%) & P. mirabilis (\%) \\
\hline Amikacin & 96.5 & 74.9 & 51.4 \\
Gentamycin & 79.3 & 63.8 & 10.9 \\
Cephalothin & 11.8 & 12.7 & 12.5 \\
Cefuroxime & 41.1 & 17.5 & 20.0 \\
Cefoxitin & 81.8 & 38.0 & 78.2 \\
Ceftazidime & 48.0 & 26.0 & 25.0 \\
Ceftriaxone & 45.7 & 27.4 & 21.8 \\
Cefepime & 47.8 & 31.3 & 23.2 \\
Aztreonam & 47.6 & 25.0 & 38.1 \\
Ampicillin & 15.6 & 0.5 & 14.0 \\
Amoxcyline-clavunate & 34.1 & 19.2 & 19.0 \\
Piptazobactam & 82.7 & 33.5 & 76.0 \\
Trimethoprim-Sulfamethoxazole & 38.3 & 33.7 & 15.8 \\
Nitrofurantoin & 82.5 & 22.4 & 0.0 \\
Ciprofloxacin & 40.8 & 31.0 & 13.1 \\
Levofloxacin & 45.0 & 35.9 & 13.1 \\
\hline
\end{tabular}

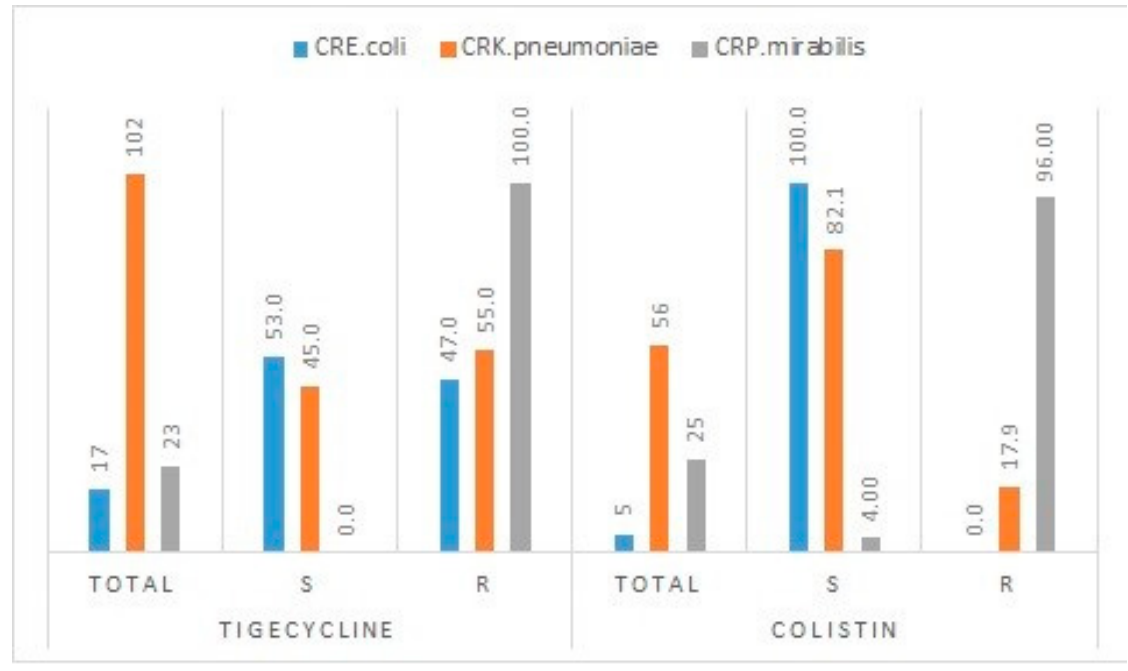

Figure 1. Tigecycline and colistin resistance in carbapenem-resistant E. coli, K. pneumoniae, and $P$. mirabilis (total in absolute numbers, and individual resistance in percentages).

\section{Discussion}

Antimicrobial resistance is prevalent worldwide, and the mortality due to drugresistant infection is predicted to reach 10 million annually by 2050 [18]. The current treatment options for drug-resistant infections are becoming less effective, thereby leading to treatment failures, increasing treatment cost, and endangering life [1]. Antimicrobial resistance, particularly carbapenem resistance in Gram-negative bacteria, is the latest challenge of immense public health importance [19]. Carbapenemase-producing Enterobacteriaceae such as KPC and New Delhi metallo- $\beta$-lactamase NDM have achieved global dissemination and pose a serious threat to human life $[8,20]$. The Kingdom of Saudi Arabia has reported a rise in carbapenem-resistant Enterobacteriaceae, mostly from Riyadh [15,21-24] and Makkah [14,25].

In the present study, K. pneumoniae, E. coli, and P. mirabilis were the major Enterobacterales isolated during the study period. These findings are in accordance with earlier studies from Saudi hospitals and countries in the MENA region [20,26,27]. These microorganisms have achieved remarkable antimicrobial resistance and have been increasingly recovered from various clinical samples [27]. 
In general, a very high prevalence $(82.1 \%)$ of MDR was observed in this study. The majority of MDR isolates belonged to K. pneumoniae and E. coli in almost equal proportions. Furthermore, this study reported $128(24.0 \%)$ isolates as extended-spectrum beta-lactamase (ESBL) producers. E. coli was the most common ESBL active, followed by K. pneumoniae. The antimicrobial resistance and prevalence of MDR or ESBL activity among bacteria are difficult to compare and depend on various local factors such as region- and countryspecific guidelines on antimicrobial use, self-medication, infection control practices, and practices affecting antimicrobial prescription and use [28]. Furthermore, study-specific factors such as analysis of clinical samples from inpatients, outpatients, or both; type of clinical sample analyzed (urine, blood, sputum, or all types of samples); treating intermediate resistance as resistant or sensitive categories; and the analysis adopted to measure various rates affect comparison across studies. A recent study evaluating the effectiveness of the Saudi Action Plan in combating antimicrobial resistance identified E. coli as the most frequently isolated Enterobacterale and the predominant bacteria for ESBL activity and MDR prevalence, followed by K. pneumoniae [29]. Similarly, another study from the neighboring country of Sudan reported E. coli and K. pneumoniae as the most commonly isolated Enterobacterales. E. coli was reported as a frequent MDR isolate, whereas ESBL activity was more frequent in K. pneumoniae [27]. These reports are in accordance with our results on the most common bacterial isolation and ESBL activity, with a slight variation in MDR prevalence among the two bacteria. A study from Addis Ababa reported a 42.1\% MDR among Enterobacteriaceae [30]; however, another recent study from Saudi Arabia reported an overall prevalence of 60\% MDR Enterobacteriaceae, with E. coli and K. pneumoniae as the most frequent isolates [31], but this prevalence was only $6.6 \%$ in the USA hospitals [32]. The analysis adopted in the present study revealed higher MDR prevalence, and this prevalence was determined for the three predominant bacteria and not for all Enterobacterales isolates reported during the study period. The extended-spectrum penicillin and cephalosporins are safer than other antimicrobials, resulting in their wide use in clinical settings [33], which might partly explain the increased MDR prevalence and ESBL activity in these bacteria. Of the 101 isolates of P. mirabilis recovered, 95 (94\%) were MDR. Our results on the MDR prevalence are consistent with a study from India that reported a $>95 \%$ MDR rate in P. mirabilis [34]. The biofilms produced by this organism not only help to evade the lethal effect of antimicrobials but also induce bacterial changes to resist and escape host defenses $[35,36]$. The free availability of antimicrobials and non-adherence, which are frequent in Saudi Arabia, could be attributed to this increased resistance and subsequent MDR prevalence.

The MDR isolates were recovered from intensive care units at a significantly higher frequency, whereas ESBL isolates were significantly higher in winter. Other studies have reported similar results regarding the frequency of MDR in intensive care units [32,37]. The reasons for this observed phenomenon are that patients admitted to intensive care units have multiple pathologies and are frequently treated with empirical antibiotic therapy before culture and sensitivity reports compared to non-intensive care unit patients. The high frequency of ESBL isolation in winter may be a result of higher antimicrobial consumption during these months. Seasonal variation in antimicrobial resistance has been previously reported [38].

In the present study, K. pneumoniae isolates exhibited resistance rates of $62.5 \%, 62 \%$ and $58 \%$ for ertapenem, imipenem, and meropenem, respectively. E. coli isolates revealed an overall carbapenem resistance of $10.9 \%$, with the highest rate of $15.8 \%$ for imipenem. Moreover, $29.3 \%$ and $25.8 \%$ resistance to ertapenem and meropenem was observed in P. mirabilis, respectively. Ali Al Bshabshe et al. reported $65.2 \%$ and $61.7 \%$ resistance in K. pneumoniae towards ertapenem and meropenem, respectively, which is consistent with our results [39]. The slight increase in these resistance rates is because their study analyzed clinical samples from intensive care units only. In another study, $38.4 \%$ and $46.1 \%$ resistance rates to imipenem and meropenem, respectively, were noted in K. pneumoniae [40]. Carbapenem resistance is a concern as these drugs are the treatment of choice, 
particularly against ESBL-producing K. pneumoniae. These rates indicate the emergence of carbapenemase-producing strains. Timely identification of carbapenem strains is pivotal for controlling these infections in hospital settings. A previous study reported that $43.5 \%$ of antimicrobials were inappropriately prescribed; of these, broad-spectrum activity (44.6\%) and use of antimicrobials without culture (32.4\%) were the main reasons [41]. Inappropriate prescriptions when coupled with self-medication and improper antibiotic consumption may greatly enhance antimicrobial resistance. Appropriate antibiotic prescription guidelines, regulation of antibiotic dispensing by community pharmacies, and patient education are some of the vital measures in combating the menace of antimicrobial resistance.

In the present study, E. coli and K. pneumoniae presented a significant seasonal variation (winter vs. other seasons) in the resistance rates for imipenem $(2.55(1.21-5.37) ; p<0.01)$ and meropenem (2.18 (1.01-4.67); $p<0.04)$, respectively. A study from the USA covering hospitalized patients did not find significant seasonal variation in carbapenem resistance among Enterobacteriaceae [42]. Although this phenomenon needs further exploration, this variation can be explained from the fact that both antibiotic consumption and antimicrobial resistance increases in the winter season [38]. Furthermore, the present study observed approximately $30 \%$ of the Enterobacteriaceae infections in winter, which may explain the different resistance rates towards carbapenems.

Colistin and tigecycline are used against carbapenem-resistant Enterobacteriaceae (CRE). This study evaluated the antimicrobial activity of these antibiotics against carbapenemresistant (meropenem-resistant) and multidrug-resistant strains identified in this study. Tigecycline exhibited 53\% and $45 \%$ sensitivity against E. coli (CRE) and K. pneumoniae (CRE), respectively. Similarly, colistin was $100 \%$ and $82.1 \%$ effective against these two strains, respectively. Both antibiotics were ineffective against $P$. mirabilis. The treatment options for carbapenem-resistant strains are limited, that is, the current standard treatment for such infections includes tigecycline, polymyxins, fosfomycin, and aminoglycosides, which are administered alone or in combination with other antibiotics, the latter revealing better outcomes. An increase in tigecycline sensitivity from $76 \%$ to $82 \%$ against E. coli (CRE) and from 33\% to 50\% for K. pneumoniae (CRE) has been reported in Riyadh. The same study reported a decline in colistin sensitivity from $97 \%$ to $86 \%$ against $E$. coli (CRE) and from $80 \%$ to $76 \%$ against K. pneumoniae (CRE) [43]. The observations from Riyadh complement our findings on these two antibiotics, with subtle variations. Biofilms and intrinsic resistance to colistin and tigecycline are prominent features of $P$. mirabilis, which explains its resistance towards these antibiotics [44].

The major strength of this study is that it is the first exclusive report on the resistance pattern in Enterobacterales from this region. Furthermore, the study analyzed the recent data of 2019, which is the second strength of the study. One limitation of this study is that it included data from only one referral center out of two operating in the capital city of the Aljouf region, thereby limiting the generalizability of the results; however, the study hospital is the oldest in the region and continues to process the majority of clinical samples in addition to those received from other hospitals. Another limitation is the lack of molecular analysis of this acquired resistance.

\section{Conclusions}

The study concludes that a high prevalence of MDR and carbapenem resistance exist in the study area. Intensive care units majorly contribute to the prevalence of MDR. Seasonal variations in ESBL-producing bacteria and carbapenem resistance were noted. High tigecycline resistance was noted, which has an immense impact on treatment options and patient outcomes. Antimicrobial resistance rates are worrying and require immediate action at the institutional and public health levels. Strict infection control practices and antimicrobial prescription guidelines need to be observed in the intensive care units to counter antimicrobial resistance. The seasonal variation in carbapenem resistance should guide empirical antibiotic therapy in this hospital. Identification of the molecular basis of 
the observed resistance and exploring the reasons for the seasonal variation are potential areas for future research.

\section{Material and Methods}

The Aljouf region has three governorates of Sakaka, Qurayyat, and Dumat Al-Jandal. The total population of the Aljouf region is 520,737 as per the 2018 census. The Sakaka governorate is the capital city of the Aljouf region with two specialist hospitals of 300 beds each. The study was conducted in one of the two specialist hospitals that serve the population of this region. This hospital is the first specialist hospital built in the region. This retrospective hospital-based study analyzed the data on culture and antibacterial reports of all non-duplicate Enterobacteriaceae isolated from 1 January 2019 to 31 December 2019. The BD Phoenix system (BD Diagnostics, Sparks, MD, USA) processed all the samples and generated a sensitivity report.

The antibiotics used to assess bacterial susceptibility were imipenem, meropenem, ertapenem, amikacin, gentamycin, aztreonam, ampicillin, augmentin, piperacillin/tazobactam, cephalothin, cefoxitin, cefuroxime, ceftazidime, ceftriaxone, cefepime, nitrofurantoin, ciprofloxacin, levofloxacin, tigecycline, colistin, and trimethoprim/sulfamethoxazole. Intermediate resistance presented by the bacterial isolates was interpreted as being resistant. Magiorakos et al.'s criteria were used for classification of antimicrobial resistance into multidrug resistance (MDR), extensive drug resistance (XDR), and pan drug resistance (PDR) [45]. Multidrug resistance (MDR) is defined as when a bacterium is 'non-susceptible to $\geq 1$ agent in $\geq 3$ antimicrobial categories'. Extensive drug resistance (XDR) is defined as when a bacterium is 'non-susceptible to $\geq 1$ agent in all but $\leq 2$ categories (i.e., bacterial isolates remain susceptible to only one or two categories), while pan drug resistance (PDR) is defined as when a bacterium is 'non-susceptible to all antimicrobial agents listed' [45]. Moreover, carbapenem-resistant Enterobacteriaceae (ER) was defined according to the revised document of the Centers for Disease Control and Prevention CR-2015 [46]. The bacterial antimicrobial susceptibility results were interpreted using breakpoints established by the Clinical Laboratory Standards Institute (CLSI) [47]. Information on the phenotypic characterization of ESBL provided by the BD Phoenix system was also recorded. Furthermore, the study collected data on the type of specimen, specimen collection date, information of different units referring the sample, and age and gender of patients.

\section{Statistics}

Statistical Package for Social Sciences (SPSS) version 21.0 for Windows (SPSS, Inc., Chicago, IL, USA) was used for data analysis. The entries were manually verified for accuracy. The frequencies and percentages of Enterobacteriaceae distribution and MDR isolates were calculated. Additionally, the association between sociodemographic and distribution characteristics of Enterobacteriaceae was assessed using the chi-square test with or without Yates correction. Fisher's exact test was performed whenever necessary. Furthermore, the distribution characteristics of multidrug-resistant strains and various carbapenem-resistant isolates vis-à-vis sociodemographic risk factors such as sex, age groups, seasonality, referring unit, and type of sample were determined by odds ratios with $95 \%$ confidence intervals. Statistical significance was set at $p<0.05$.

Author Contributions: Conceptualization, A.B.; Data curation, B.T.; Formal analysis, A.B.; Methodology, A.B.; Writing—original draft, B.T.; Writing—review and editing, A.B. All authors have read and agreed to the published version of the manuscript.

Funding: This work did not receive any funding.

Institutional Review Board Statement: Jouf University's Local Committee on Bio-Ethics approved the research proposal vide 03-04/41, dated 6 January 2020. Informed consent is a standard practice ensured at the time of specimen collection in this hospital. For patients admitted to the intensive care unit, informed consent of the legal guardian is recorded. Research was performed in accordance with relevant guidelines/regulations and in accordance with the Declaration of Helsinki. This 
study analyzed the existing data on antibiograms. The patients' identifiable data points like name, medical record number, and specimen number were removed at the time of data collection to delink identification.

Informed Consent Statement: Informed consent was obtained from all subjects involved in the study.

Data Availability Statement: The datasets generated and/or analyzed during the current study are available from the corresponding author on reasonable request. The dataset will be uploaded in the relevant repository after acceptance.

Acknowledgments: The authors thanks Tarek El-Metwally Dabah for his continuous encouragement for this research.

Conflicts of Interest: The authors declare no conflict of interest.

\section{References}

1. Shrivastava, S.R.; Shrivastava, P.S.; Ramasamy, J. World health organization releases global priority list of antibiotic-resistant bacteria to guide research, discovery, and development of new antibiotics. J. Med. Soc. 2018, 32, 76. [CrossRef]

2. Weiner, L.M.; Webb, A.K.; Limbago, B.; Dudeck, M.A.; Patel, J.; Kallen, A.J.; Edwards, J.R.; Sievert, D.M. AntimicrobialResistant Pathogens Associated with Healthcare-Associated Infections: Summary of Data Reported to the National Healthcare Safety Network at the Centers for Disease Control and Prevention, 2011-2014. Infect. Control Hosp. Epidemiol. 2016, 37, 1288-1301. [CrossRef] [PubMed]

3. Tumbarello, M.; Viale, P.; Viscoli, C.; Trecarichi, E.M.; Tumietto, F.; Marchese, A.; Spanu, T.; Ambretti, S.; Ginocchio, F.; Cristini, F.; et al. Predictors of mortality in bloodstream infections caused by Klebsiella pneumoniae carbapenemase-producing K. pneumoniae: Importance of combination therapy. Clin. Infect. Dis. 2012, 55, 943-950. [CrossRef]

4. Tzouvelekis, L.; Markogiannakis, A.; Psichogiou, M.; Tassios, P.; Daikos, G. Carbapenemases in Klebsiella pneumoniae and other Enterobacteriaceae: An evolving crisis of global dimensions. Clin. Microbiol. Rev. 2012, 25, 682-707. [CrossRef] [PubMed]

5. Swaminathan, M.; Sharma, S.; Blash, S.P.; Patel, G.; Banach, D.B.; Phillips, M.; LaBombardi, V.; Anderson, K.F.; Kitchel, B.; Srinivasan, A.; et al. Prevalence and Risk Factors for Acquisition of Carbapenem-Resistant Enterobacteriaceae in the Setting of Endemicity. Infect. Control Hosp. Epidemiol. 2013, 34, 809-817. [CrossRef]

6. Xu, Y.; Gu, B.; Huang, M.; Liu, H.; Xu, T.; Xia, W.; Wang, T. Epidemiology of carbapenem resistant Enterobacteriaceae (CRE) during 2000-2012 in Asia. J. Thorac. Dis. 2015, 7, 376-385. [CrossRef] [PubMed]

7. World Health Organization. Antimicrobial Resistance: Global Report on Surveillance; World Health Organization: Geneva, Switzerland, 2014.

8. Tängdén, T.; Giske, C. Global dissemination of extensively drug-resistant carbapenemase-producing E nterobacteriaceae: Clinical perspectives on detection, treatment and infection control. J. Intern. Med. 2015, 277, 501-512. [CrossRef] [PubMed]

9. Hussein, K.; Raz-Pasteur, A.; Finkelstein, R.; Neuberger, A.; Shachor-Meyouhas, Y.; Oren, I.; Kassis, I. Impact of carbapenem resistance on the outcome of patients' hospital-acquired bacteraemia caused by Klebsiella pneumoniae. J. Hosp. Infect. 2013, 83, 307-313. [CrossRef]

10. Al-Zahrani, I.A.; Alasiri, B.A. The emergence of carbapenem-resistant Klebsiella pneumoniae isolates producing OXA-48 and NDM in the Southern (Asir) province, Saudi Arabia. Saudi Med. J. 2018, 39, 23-30. [CrossRef] [PubMed]

11. El Ghany, M.A.; Sharaf, H.; Al-Agamy, M.H.; Shibl, A.; Hill-Cawthorne, G.A.; Hong, P.-Y. Genomic characterization of NDM-1 and 5, and OXA-181 carbapenemases in uropathogenic Escherichia coli isolates from Riyadh, Saudi Arabia. PLoS ONE 2018, 13, e0201613. [CrossRef]

12. Saeed, W.M.; Ghanem, S.; El Shafey, H.M.; Manzoor, N. Assessment of antimicrobial resistance patterns in Escherichia coli isolated from clinical samples in Madinah, Saudi Arabia. Afr. J. Microbiol. Res. 2018, 12, 321-326.

13. Alotaibi, F.E.; Bukhari, E.E.; Al-Mohizea, M.M.; Hafiz, T.; Essa, E.B.; AlTokhais, Y.I. Emergence of carbapenem-resistant Enterobacteriaceae isolated from patients in a university hospital in Saudi Arabia. Epidemiology, clinical profiles and outcomes. J. Infect. Public Health 2017, 10, 667-673. [CrossRef]

14. Khan, M.A.; Faiz, A. Frequency of carbapenemase producing Klebsiella pneumoniae in Makkah, Saudi Arabia. J. Microbiol. Infect. Dis. 2016, 6, 121-127.

15. Al-Agamy, M.H.; Aljallal, A.; Radwan, H.H.; Shibl, A.M. Characterization of carbapenemases, ESBLs, and plasmid-mediated quinolone determinants in carbapenem-insensitive Escherichia coli and Klebsiella pneumoniae in Riyadh hospitals. J. Infect. Public Health 2018, 11, 64-68. [CrossRef]

16. Gupta, N.; Limbago, B.M.; Patel, J.B.; Kallen, A.J. Carbapenem-Resistant Enterobacteriaceae: Epidemiology and Prevention. Clin. Infect. Dis. 2011, 53, 60-67. [CrossRef] [PubMed]

17. Di Tella, D.; Tamburro, M.; Guerrizio, G.; Fanelli, I.; Sammarco, M.L.; Ripabelli, G. Molecular Epidemiological Insights into Colistin-Resistant and Carbapenemases-Producing Clinical Klebsiella pneumoniae Isolates. Infect. Drug Resist. 2019, 12, 3783-3795. [CrossRef] [PubMed]

18. Shahpawee, N.S.; Chaw, L.L.; Muharram, S.H.; Goh, H.P.; Hussain, Z.; Ming, L.C. University Students' Antibiotic Use and Knowledge of Antimicrobial Resistance: What Are the Common Myths? Antibiotics 2020, 9, 349. [CrossRef] [PubMed] 
19. Jacob, J.T.; Klein, E.; Laxminarayan, R.; Beldavs, Z.; Lynfield, R.; Kallen, A.J.; Ricks, P.; Edwards, J.; Srinivasan, A.; Fridkin, S.; et al. Vital signs: Carbapenem-resistant Enterobacteriaceae. MMWR Morb. Mortal. Wkly. Rep. 2013, 62, 165.

20. Alotaibi, F. Carbapenem-Resistant Enterobacteriaceae: An update narrative review from Saudi Arabia. J. Infect. Public Health 2019, 12, 465-471. [CrossRef]

21. Zaman, T.U.; AlDrees, M.; Al Johani, S.M.; Alrodayyan, M.; Aldughashem, F.A.; Balkhy, H.H. Multi-drug carbapenem-resistant Klebsiella pneumoniae infection carrying the OXA-48 gene and showing variations in outer membrane protein 36 causing an outbreak in a tertiary care hospital in Riyadh, Saudi Arabia. Int. J. Infect. Dis. 2014, 28, 186-192. [CrossRef]

22. Shibl, A.; Al-Agamy, M.; Memish, Z.; Senok, A.; Khader, S.A.; Assiri, A. The emergence of OXA-48- and NDM-1-positive Klebsiella pneumoniae in Riyadh, Saudi Arabia. Int. J. Infect. Dis. 2013, 17, e1130-e1133. [CrossRef]

23. Marie, M.A.; John, J.; Krishnappa, L.G.; Gopalkrishnan, S. Molecular characterization of the $\beta$-lactamases in Escherichia coli and Klebsiella pneumoniae from a tertiary care hospital in Riyadh, Saudi Arabia. Microbiol. Immunol. 2013, 57, 805-810. [CrossRef] [PubMed]

24. Al-Agamy, M.H.; Shibl, A.M.; Elkhizzi, N.A.; Meunier, D.; Turton, J.F.; Livermore, D.M. Persistence of Klebsiella pneumoniae clones with OXA-48 or NDM carbapenemases causing bacteraemias in a Riyadh hospital. Diagn. Microbiol. Infect. Dis. 2013, 76, 214-216. [CrossRef]

25. Faidah, H.S.; Momenah, A.M.; El-Said, H.M.; Barhameen, A.A.A.; Ashgar, S.S.; Johargy, A.; Elsawy, A.; Almalki, W.; Al Qurashi, S. Trends in the Annual Incidence of Carbapenem Resistant among Gram Negative Bacilli in a Large Teaching Hospital in Makah City, Saudi Arabia. J. Tuberc. Res. 2017, 5, 229-236. [CrossRef]

26. Yezli, S.; Shibl, A.M.; Memish, Z.A. The molecular basis of $\beta$-lactamase production in Gram-negative bacteria from Saudi Arabia. J. Med. Microbiol. 2015, 64, 127-136. [CrossRef]

27. Moglad, E.H. Antibiotics Profile, Prevalence of Extended-Spectrum Beta-Lactamase (ESBL), and Multidrug-Resistant Enterobacteriaceae from Different Clinical Samples in Khartoum State, Sudan. Int. J. Microbiol. 2020, 2020. [CrossRef] [PubMed]

28. Ayukekbong, J.A.; Ntemgwa, M.; Atabe, A.N. The threat of antimicrobial resistance in developing countries: Causes and control strategies. Antimicrob. Resist. Infect. Control 2017, 6, 1-8. [CrossRef]

29. Alabdullatif, M.; Alrehaili, J. Three Years of Evaluation to Determine Reduction of Antibiotic Resistance in Gram-Negative Bacteria by the Saudi National Action Plan. Infect. Drug Resist. 2020, 13, 3657-3667. [CrossRef] [PubMed]

30. Bitew, A.; Tsige, E. High Prevalence of Multidrug-Resistant and Extended-Spectrum $\beta$-Lactamase-Producing Enterobacteriaceae: A Cross-Sectional Study at Arsho Advanced Medical Laboratory, Addis Ababa, Ethiopia. J. Trop. Med. 2020, 2020. [CrossRef]

31. Alkofide, H.; Alhammad, A.M.; Alruwaili, A.; Aldemerdash, A.; Almangour, T.; Alsuwayegh, A.; Almoqbel, D.; Albati, A.; Alsaud, A.; Enani, M. Multidrug-Resistant and Extensively Drug-Resistant Enterobacteriaceae: Prevalence, Treatments, and Outcomes-A Retrospective Cohort Study. Infect. Drug Resist. 2020, 13, 4653-4662. [CrossRef]

32. Gupta, V.; Ye, G.; Olesky, M.; Lawrence, K.; Murray, J.; Yu, K. National prevalence estimates for resistant Enterobacteriaceae and Acinetobacter species in hospitalized patients in the United States. Int. J. Infect. Dis. 2019, 85, 203-211. [CrossRef] [PubMed]

33. Bush, K.; Bradford, P.A. $\beta$-Lactams and $\beta$-lactamase inhibitors: An overview. Cold Spring Harb. Perspect. Med. 2016, 6, a025247. [CrossRef]

34. Pal, N.; Sharma, N.; Sharma, R.; Hooja, S.; Maheshwari, R.K. Prevalence of multidrug (MDR) and extensively drug resistant (XDR) Proteus species in a tertiary care hospital, India. Int. J. Curr. Microbiol. Appl. Sci. 2014, 3, $243-252$.

35. Høiby, N.; Bjarnsholt, T.; Givskov, M.; Molin, S.; Ciofu, O. Antibiotic resistance of bacterial biofilms. Int. J. Antimicrob. Agents 2010, 35, 322-332. [CrossRef] [PubMed]

36. Stewart, P.S. Mechanisms of antibiotic resistance in bacterial biofilms. Int. J. Med. Microbiol. 2002, 292, 107-113. [CrossRef] [PubMed]

37. Basak, S.; Singh, P.; Rajurkar, M. Multidrug Resistant and Extensively Drug Resistant Bacteria: A Study. J. Pathog. 2016, 2016, 1-5. [CrossRef] [PubMed]

38. Ramsey, E.G.; Royer, J.; Bookstaver, P.B.; Justo, J.A.; Kohn, J.; Albrecht, H.; Al-Hasan, M.N. Seasonal variation in antimicrobial resistance rates of community-acquired Escherichia coli bloodstream isolates. Int. J. Antimicrob. Agents 2019, 54, 1-7. [CrossRef]

39. Al Bshabshe, A.; Al-Hakami, A.; AlShehri, B.; Al-Shahrani, K.A.; AlShehri, A.A.; Al Shahrani, M.B.; Assiry, I.; Joseph, M.R.; Alkahtani, A.M.; Hamid, M.E. Rising Klebsiella pneumoniae Infections and Its Expanding Drug Resistance in the Intensive Care Unit of a Tertiary Healthcare Hospital, Saudi Arabia. Cureus 2020, 12. [CrossRef]

40. Al-Zalabani, A.; AlThobyane, O.A.; AlShehri, A.H.; AlRehaili, A.O.; Namankani, M.O.; Aljafri, O.H. Prevalence of Klebsiella pneumoniae Antibiotic Resistance in Medina, Saudi Arabia, 2014-2018. Cureus 2020, 12. [CrossRef]

41. Alsaleh, N.A.; Al-Omar, H.A.; Mayet, A.Y.; Mullen, A.B. Evaluating the appropriateness of carbapenem and piperacillintazobactam prescribing in a tertiary care hospital in Saudi Arabia. Saudi Pharm. J. 2020, 28, 1492-1498. [CrossRef] [PubMed]

42. Gupta, V.; Ye, G.; Olesky, M.; Lawrence, K.; Murray, J.; Yu, K. Trends in resistant Enterobacteriaceae and Acinetobacter species in hospitalized patients in the United States: 2013-2017. BMC Infect. Dis. 2019, 19, 1-9. [CrossRef] [PubMed]

43. Aldawsari, A.; Tawfik, K.; Al-Zaagi Sr, I. Antimicrobial-Resistant Bacteria and Prescription of Antibiotics at a Tertiary Care Hospital in Riyadh, Saudi Arabia. Cureus 2020, 12, e12098. [CrossRef] [PubMed]

44. Qin, S.; Qi, H.; Zhang, Q.; Zhao, D.; Liu, Z.-Z.; Tian, H.; Xu, L.; Xu, H.; Zhou, M.; Feng, X.; et al. Emergence of Extensively Drug-Resistant Proteus mirabilis Harboring a Conjugative NDM-1 Plasmid and a Novel Salmonella Genomic Island 1 Variant, SGI1-Z. Antimicrob. Agents Chemother. 2015, 59, 6601-6604. [CrossRef] [PubMed] 
45. Magiorakos, A.-P.; Srinivasan, A.; Carey, R.T.; Carmeli, Y.; Falagas, M.T.; Giske, C.T.; Harbarth, S.; Hindler, J.T.; Kahlmeter, G.; Olsson-Liljequist, B.; et al. Multidrug-resistant, extensively drug-resistant and pandrug-resistant bacteria: An international expert proposal for interim standard definitions for acquired resistance. Clin. Microbiol. Infect. 2012, 18, 268-281. [CrossRef] [PubMed]

46. Centers for Disease Control and Prevention. Facility Guidance for Control of Carbapenem-Resistant Enterobacteriaceae (CRE) November 2015 Update CRE Toolkit; Centers for Disease Control and Prevention: Atlanta, GA, USA; United States Department of Health and Human Services: Washington, DC, USA, 2016.

47. Wayne, P.A. Clinical and laboratory standards institute. Performance standards for antimicrobial susceptibility testing. Inform. Suppl. 2011, 31, 100-121. 